\title{
Contamination of blood cultures during venepuncture: fact or myth?
}

\author{
Eyal Shahar, Bat-Sheva Wohl-Gottesman and Louis Shenkman
}

\author{
Department of Medicine 'C', Meir General Hospital, Sapir Medical Center, Kfar-Saba, and the \\ Department of Medicine, the Sackler School of Medicine, Tel-Aviv University, Tel-Aviv, Israel
}

\begin{abstract}
Summary: Contamination of blood cultures is believed to occur mainly during the venepuncture procedure. Consequently, meticulous preparation of the venepuncture site is widely recommended. To determine whether the contamination rate is indeed affected by the quality of the antiseptic procedure at the venepuncture site, 181 paired cultures were collected from 176 patients during a 6-month period after either strict antiseptic cleansing of skin with alcohol followed by povidone-iodine, or after brief disinfection with alcohol alone.

The contamination rate was not influenced by the antiseptic procedure, and corresponded to the accepted percentage reported in most other studies. Eight false positive cultures (4.4\%) were obtained after strict antisepsis of the skin and $6(3.3 \%)$ after short simple cleansing with alcohol $(P=0.39)$. Our results suggest that contamination of blood cultures may not be related to the venepuncture procedure regardless of the antiseptic technique used - but may be due to later stages of laboratory handling and processing of the specimens. Review of the literature has provided further indirect evidence to support this conclusion.
\end{abstract}

\section{Introduction}

Between $2 \%$ to $5 \%$ of all blood cultures ${ }^{1-3}$ and at least $25 \%$ of positive ones ${ }^{1}$ are eventually designated as falsely positive, representing contamination rather than true bacteraemia. Because frequently recovered contaminants - such as coagulase-negative staphylococci or Bacillus species - are also capable of causing life-threatening infections, ${ }^{4-7}$ it is often difficult to decide whether a positive blood culture is clinically important or represents pseudobacteraemia. Occasionally, a cluster of pseudobacteraemias may even cause a pseudoepidemic of nosocomial infection. ${ }^{8,9}$ Therefore, continuous efforts are being made to reduce the false positive rate of blood cultures.

Contamination of blood cultures may occur during any stage of the process, ${ }^{9}$ from manufacturing the media ${ }^{10}$ through laboratory handling and processing of the specimens. ${ }^{11-14}$ Nevertheless, it is widely believed that the majority of contamination occurs during the venepuncture procedure. ${ }^{15-17}$ The common explanation suggests that insertion of the needle through poorly disinfected skin causes contamination of the needle edge by indigenous

Correspondence: E. Shahar, M.D., Department of Medicine, P.O. Box 6052 Tel-Aviv 61060, Israel Accepted: 4 June 1990 microbial flora, which is inoculated thereafter into the syringe while blood is withdrawn. Consequently, standard textbooks, ${ }^{18}$ clinical microbiology manuals, ${ }^{16,19}$ and several review articles ${ }^{3,15,17}$ recommend strict antiseptic preparation of the venepuncture site prior to obtaining blood cultures. These recommendations have become a routine practice of house officers and venepuncture teams.

Surprisingly, after careful search of the literature, we could find little scientific evidence to verify this long-standing belief. The following prospective study was undertaken to determine whether the contamination rate of blood cultures is indeed affected by the quality of the antiseptic procedure at the venepuncture site.

\section{Methods}

During a 6-month period all blood cultures ordered by attending physicians in our internal medicine ward were obtained by the house officers according to the following protocol. As recently recommended, ${ }^{3}$ two blood samples were collected from patients suspected to have septicaemia, usually several hours apart. Occasionally, when infective endocarditis was a likely diagnosis, four samples 
were collected. Blood samples were drawn from antecubital veins using 21-gauge needles and disposable syringes. From each patient, the two samples were collected, handled and processed in an identical manner except for venepuncture site preparation. All blood cultures included in this study were obtained before administration of antibiotic therapy or at least $\mathbf{4 8}$ hours after discontinuation of such treatment.

One blood culture was obtained after strict antiseptic cleansing, recommended by most authors. ${ }^{3,15-19}$ This consisted of application of $70 \%$ alcohol for one minute followed by application of povidone-iodine solution ( $1 \%$ available iodine) for an additional minute. The latter was carried out using a sterile applicator in a concentric manner. Thereafter, a venepuncture was performed without palpating the vein again.

The other blood culture was collected after 'routine' preparation of the venepuncture site similar to skin disinfection performed for any other blood sampling. The skin was cleansed briefly by swabbing 3 to 5 times with $70 \%$ alcohol, and venepuncture was performed within 5 to 10 seconds thereafter. Whenever four blood cultures were obtained, two were collected by each procedure.

In each venepuncture 6 to $10 \mathrm{ml}$ of blood were drawn into a 10-ml disposable syringe. After replacing the needle and swabbing the rubber stoppers of blood culture bottles with $70 \%$ alcohol, equal volumes of the sample were inoculated at the bedside into an aerobic BACTEC $6 \mathrm{~B}$ bottle and an anaerobic BACTEC 7D bottle (Johnston Laboratories, Towson, Maryland). All blood cultures underwent standard incubation and processing in the Department of Clinical Microbiology of our hospital, using a BACTEC 460 radiometric analyser (Johnston Laboratories). ${ }^{20}$ Unless specifically requested, blood cultures were incubated for 7 days, and declared negative if no growth was detected by then.

A blood culture was considered positive if an organism was isolated from a single venepuncture in either or both media. The definition of false positive results was based on clinical judgement, following previously described guidelines..$^{21,22}$ In order to avoid any possible bias, the Clinical Microbiology department was not notified of the ongoing study until its completion.

Statistical analysis included comparing proportions by the McNemar's test with a correction for continuity. Student's $t$-test for unpaired samples was used to compare means.

\section{Results}

During the 6-month study period, blood cultures were obtained from 176 patients. Two blood cultures were collected from each of 171 patients, and 4 cultures were obtained from the remaining five. In all, 362 blood cultures were collected, half after strict antiseptic cleansing of the venepuncture site (alcohol followed by povidone-iodine) and half after routine swabbing with alcohol.

Twenty-six of the 362 cultures (7.2\%) were positive, of which $12(3.3 \%)$ were considered to be true positive cultures, while $14(3.9 \%)$ were judgee to be contaminated. Of note, isolates judged to be contaminants were detected by the laboratory after a longer period of incubation (mean 6.2 days; s.d. 2.9) as compared with true pathogens (mean 3.5 days; s.d. 0.6) $(P<0.001)$.

Table I depicts the distribution of the contaminants between the two methods of skin preparation, and the organisms recovered. The contamination rate was similar in both groups: 8 false positive cultures $(4.4 \%)$ were reported after strict antiseptic cleansing, and $6(3.3 \%)$ after

Table I Distribution of blood culture contaminants according to method of skin preparation

\begin{tabular}{|c|c|c|c|}
\hline Organism & $\begin{array}{r}\text { No. of } \\
\text { Strict antisepsis* } \\
(n=181)\end{array}$ & $\begin{array}{l}\text { Routine preparation } \\
\qquad(\%=181)\end{array}$ & $P$ value \\
\hline $\begin{array}{l}\text { Staphylococcus } \\
\text { epidermidis }\end{array}$ & 2 & 2 & \\
\hline Bacillus species & 4 & 3 & \\
\hline$\alpha$-Streptococcus & 1 & - & \\
\hline Enterobacter & - & 1 & \\
\hline Diphtheroid & 1 & - & \\
\hline Total & $8(4.4)$ & $6(3.3)$ & NSt \\
\hline
\end{tabular}

*Strict antisepsis = alcohol followed by povidone-iodine; Routine preparation $=$ alcohol swabbing (see text for details). †NS denotes not significant. 
routine cleansing with alcohol $(P=0.39$; not significant).

Table II and Table III present the data on the isolation pattern of contaminants and true positive blood cultures, respectively, according to the culture media. While all of the true pathogens were isolated in both media, most contaminants were isolated either in aerobic or anaerobic culture media, but not in both.

\section{Discussion}

Our data failed to show any effect of the antiseptic procedure at the venepuncture site on the false positive rate of blood cultures. Careful skin antisepsis did not reduce the expected contamination rate, while 'careless' disinfection did not increase it. There may be two alternative interpretations for this finding: either that both antiseptic protocols used in this study were of comparable quality or that the contamination was not associated at all with the venepuncture act.

There have been numerous reports on the quality of different disinfectants, of which iodine solutions are well-established preparations. ${ }^{23}$ Sequential or combined application of alcohol and iodine, with proper contact time with the skin, has been recom- mended by most authors, ${ }^{3,15-19}$ and has been proven to be highly efficient. ${ }^{24-26}$ Although $70 \%$ alcohol alone is a commonly used skin antiseptic, it has been questioned whether its application for a few seconds, as performed in everyday practice, has any important value at all. ${ }^{27}$ Sterilization is not achieved instantly and adequate contact time is especially crucial with volatile products. ${ }^{28}$ Moreover, complete eradication of skin bacteria does not occur even after application of alcohol for longer periods of time. ${ }^{29,30}$ Thus, we cannot attribute our results to similar qualities of the antiseptic procedures. Furthermore, if both a highly sterile skin preparation and a questionable antiseptic technique yield similar false positive rates, one should conclude that the contamination was not associated at all with the venepuncture procedure.

Our conclusion seems to contradict a longstanding, yet unproven belief shared by both physicians and clinical microbiologists. However, review of the literature on blood cultures revealed indirect evidence to support this conclusion.

Several independent studies, ${ }^{31-37}$ designed to compare the efficacy of different blood culture media, provide the first line of evidence. All of these investigations have shared similar methodology: each blood sample, obtained by one venepuncture, was divided between two different blood culture

Table II Isolation pattern of contaminants according to culture media

\begin{tabular}{lccc}
\hline Organism & $\begin{array}{c}\text { Aerobic media } \\
\text { only }\end{array}$ & $\begin{array}{c}\text { No. of cultures } \\
\text { Anaerobic media } \\
\text { only }\end{array}$ & $\begin{array}{c}\text { Both } \\
\text { media }\end{array}$ \\
\hline $\begin{array}{l}\text { Staphylococcus } \\
\text { epidermidis }\end{array}$ & 2 & 2 & - \\
$\begin{array}{l}\text { Bacillus species } \\
\alpha-S t r e p t o c o c c u s\end{array}$ & 3 & 2 & $2^{*}$ \\
Enterobacter & 1 & - & - \\
Diphtheroid & 1 & - & - \\
Total & - & 1 & - \\
\hline
\end{tabular}

*Isolates were recovered after more than 3 weeks of incubation.

Table III Isolation pattern of true positive cultures according to culture media

\begin{tabular}{lcccl}
\hline & \multicolumn{4}{c}{ No. of cultures } \\
Organism & $\begin{array}{c}\text { Aerobic } \\
\text { only }\end{array}$ & $\begin{array}{c}\text { Anaerobic } \\
\text { only }\end{array}$ & $\begin{array}{c}\text { Both } \\
\text { media }\end{array}$ & $\begin{array}{l}\text { Clinical } \\
\text { diagnosis }\end{array}$ \\
\hline E. coli & - & - & $4^{*}$ & Urosepsis \\
T-Streptococcus & - & - & $4 \dagger$ & Endocarditis \\
Enterococcus & - & - & 2 & Urosepsis \\
Klebsiella & - & - & 2 & Urosepsis \\
Total & - & - & 12 & \\
\hline
\end{tabular}

\footnotetext{
*From two patients. †All isolates were recovered from one patient.
} 
media. Careful observation of the reported results of these trials reveals a consistent trend: while classical pathogenic bacteria, representing true bacteraemias, were usually recovered from both media, classical contaminants, in most instances, were recovered from either one or the other of the two tested media. A similar pattern is apparent in another study ${ }^{38}$ which compared bedside inoculation of half a blood sample versus in-laboratory inoculation of the other half, and in a recent study ${ }^{39}$ designed to compare the Signal system for the detection of bacteraemias with the conventional one. Again, true pathogens were recovered in both halves of a sample, while contaminants were usually isolated from only one of the two.

This consistent discrepancy in isolation pattern is difficult to settle if one assumes contamination to occur during the venepunctures. Had it been the case, contaminants - just like true blood-borne pathogens - would have been present in the syringe immediately after the venepuncture, and would have been expected to be inoculated into both bottles. Alternatively, we suggest that contaminants, unlike true pathogens, were not present in the blood samples shortly after the venepunctures, but were introduced at a later stage into one inoculated half. Therefore, they were usually isolated from only one bottle of a simultaneously inoculated pair.

Our study shows the very same phenomenon: although we did not use two different commercial media, each blood sample was divided between both aerobic and anaerobic culture bottles. The latter is equally useful for the detection of many aerobic organisms. ${ }^{5,40}$ As our results demonstrate, all isolates of true bacteraemias were recovered from both bottles (Table III), while the majority of contaminants were recovered from only one of a simultaneously inoculated pair (Table II). Only twice was a contaminant (Bacillus species) isolated from the aerobic-anaerobic pair (Table II), and that occurred in one patient. It seems highly unlikely to attribute these isolates to coincidental contamination by the same organisms during two separate venepunctures after two different antiseptic procedures.

Further supporting evidence is derived from a well-known characteristic of contaminated blood cultures that perhaps has not received sufficient attention. Delayed detection of bacterial growth is far more common for contaminants than for true pathogens. ${ }^{3}$ This phenomenon was observed in our study as well as in others. ${ }^{1,32,33}$ If contamination occurs during venepuncture, contaminants and true pathogens are inoculated into blood culture bottles at the same time and should be detected after similar incubation intervals. The fact that contaminants are detected later than true pathogens by the microbiology laboratories suggests that contaminants are introduced at a later stage of the process.

Could a difference in the inoculum size account for this discrepancy between true pathogens and contaminants - namely, large inoculum of true bacteraemias versus small one of skin contamination? This explanation should be rejected on two grounds: first, there is sufficient experimental data showing that most bacteraemias are of low order of magnitude (i.e., few colony-forming units per millilitre of blood) ${ }^{40,41}$ Second, there is no reason to assume that skin contamination, if it occurs, introduces smaller inoculum. On the contrary, since skin bacteria are present in colonies ${ }^{29}$ one would expect that incomplete disinfection would introduce large inoculum of multiple species. This is rarely the case in contaminated blood cultures.

Recently, a cohort study of coagulase-negative staphylococci infection in newborn infants ${ }^{42}$ has provided a more direct evidence against the concept of skin contamination as the source of false positive blood cultures. As part of that study, blood cultures were collected from infants without apparent infection. Following skin antisepsis, and immediately before the venepuncture, a sterile swab was rolled over the venepuncture site and inoculated on to a blood agar plate. Although there were $4.3 \%$ of false positive blood cultures an $\$$ $2.9 \%$ of positive skin swabs, in neither case has ever occurred in the same patient.

Several methods for the detection of bacteraemias have been developed over the years, and it is evident that the contamination rate is influenced by the system applied. For example, two newly introduced methods, lysis-centrifugation and lysisfiltration techniques, are particularly prone to higher contamination rates. ${ }^{36,41,43}$ Moreover, it was shown that shifting from the conventional method of blind subcultures to the semi-automated BACTEC system has resulted in decreased contamination by Bacillus species. ${ }^{44}$

Finally, direct evidence was provided by a study of 'mock' blood cultures. ${ }^{45}$ In this study sterile broth samples were processed by the lysiscentrifugation method as if they were true blood cultures. That resulted in overall contamination rate of $22 \%$ (high above the usual percentage), definitely without any possible contribution of venepuncture procedure.

In conclusion, our results, supported by additional evidence, indicate that contamination of blood cultures is not usually related to the venepuncture procedure - regardless of the antiseptic technique used - but may be due to later stages of laboratory handling and processing of the specimens. We suggest that routine 'mock' blood cultures should be performed by departments of clinical microbiology as a measure of quality control. 
Acknowledgement

We are indebted to the Statistical Laboratory, School of Mathematics, Tel-Aviv University, for statistical analysis.

\section{References}

1. Beaman, K.D., Kasten, B.L. \& Gavan, T.L. Rate of detection of bacteremia: retrospective evaluation of 23,392 blood cultures. Cleve Clin $Q$ 1977, 44: 129-136.

2. Washington, J.A., II \& Ilstrup, D.M. Blood cultures: issues and controversies. Rev Infect Dis 1986, 8: 792-802.

3. Aronson, M.D. \& Bor, D.H. Blood cultures. Ann Intern Med 1987, 106: 246-253.

4. Ponce de Leon, S. \& Wenzel, R.P. Hospital-acquired bloodstream infections with Staphylococcus epidermidis: review of 100 cases. Am J Med 1984, 77: 639-644.

5. Kirchhoff, L.V. \& Sheagren, J.N. Epidemiology and clinical significance of blood cultures positive for coagulase-negative staphylococcus. Infect Control 1985, 6: 479-486.

6. Ihde, D.C. \& Armstrong, D. Clinical spectrum of infection due to Bacillus species. Am J Med 1973, 55: 839-845.

7. Horan, T.C., White, J.W., Jarvis, W.R. et al. Nosocomial infection surveillance, 1984. MMWR 1986, 35: 17SS-29SS.

8. Weinstein, R.A. \& Stamm, W.E. Pseudoepidemics in hospital. Lancet 1977, ii: 862-864.

9. Maki, D.G. Through a glass darkly: nosocomial pseudoepidemics and pseudobacteremias. Arch Intern Med 1980, 140: 26-28.

10. Noble, R.C. \& Reeves, S.A. Bacillus species pseudosepsis caused by contaminated commercial blood culture media. JAMA 1974, 230: 1002-1004.

11. Lynch, J.M., Anderson, A., Camacho, F.R., Winters, A.K., Hodges, G.R. \& Barnes, W.G. Pseudobacteremia caused by Clostridium sordellii. Arch Intern Med 1980, 140: 65-68.

12. Faris, H.M., Jr \& Sparling, F.F. Mima polymorpha bacteremia: false-positive cultures due to contaminated penicillinase. JAMA 1972, 219: 76-77.

13. Griffin, M.R., Miller, A.D. \& Davis, A.C. Blood culture cross contamination associated with a radiometric analyzer. J Clin Microbiol 1982, 15: 567-570.

14. Craven, D.E., Lichtenberg, D.A., Browne, K.F., Coffey, D.M., Treadwell, T.L. \& McCabe, W.R. Pseudobacteremia traced to cross-contamination by an automated blood culture analyzer. Infect Control 1984, 5: 75-78.

15. Washington, J.A., II. Blood cultures: principles and techniques. Mayo Clin Proc 1975, 50: 91-98.

16. Bartlett, R.C., Ellner, P.D. \& Washington, J.A., II. Blood cultures. In: Sherris, J.C. (ed.) Cumitech 1: Cumulative Techniques and Procedures in Clinical Microbiology. American Society for Microbiology, Washington D.C., 1974, pp. 1-6.

17. Tilton, R.C. The laboratory approach to the detection of bacteremia. Annu Rev Microbiol 1982, 36: 467-493.

18. Plorde, J.J. The diagnosis of infectious diseases. In: Braunwald, E., Isselbacher, K.J., Petersdorf, R.G., Wilson, J.D., Martin, J.B. \& Fauci, A.S. (eds) Harrison's Principles of Internal Medicine. McGraw-Hill, New York, 1987, pp. 459-466.

19. Isenberg, H.D., Washington, J.A., II, Balows, A. \& Sonnenwirth, A.C. Collection, handling, and processing of specimens. In: Lennette, E.H., Balows, A., Hausler, W.J. Jr \& Shadomy, H.J. (eds) Manual of Clinical Microbiology. American Society for Microbiology, Washington D.C., 1985, pp. 73-98.

20. Reller, L.B. Recent and innovative methods for detection of bacteremia and fungemia. Am J Med 1983, 75 (Suppl 1B): 26-30.
21. MacGregor, R.R. \& Beaty, H.N. Evaluation of positive blood cultures: guidelines for early differentiation of contaminated from valid positive cultures. Arch Intern Med 1972 , 130: 84-87.

22. Weinstein, M.P., Reller, L.B., Murphy, J.R. \& Lichtenstein, K.A. The clinical significance of positive blood cultures: a comprehensive analysis of 500 episodes of bacteremia and fungemia in adults. I. Laboratory and epidemiologic observations. Rev Infect Dis 1983, 5: 35-53.

23. Houang, E.T., Gilmore, O.J.A., Reid, C. \& Shaw, E.J. Absence of bacterial resistance to povidone iodine. $J$ Clin Pathol 1976, 29: 752-755.

24. Story, P. Testing of skin disinfectants. Br Med J 1952, 2: $1128-1130$

25. Selwyn, S. \& Ellis, H. Skin bacteria and skin disinfection reconsidered. Br Med J 1972, 1: 136-140.

26. Champagne, S., Fussell, S. \& Scheifele, D. Evaluation of skin antisepsis prior to blood culture in neonates. Infect Control 1984, 5: 489-491.

27. Dann, T.C. Routine skin preparation before injection: an unnecessary procedure. Lancet 1969, ii: 96-97.

28. Favero, M.S. Sterilization, disinfection, and antisepsis in the hospital. In: Lennette, E.H., Balows, A., Hausler, W.J. Jr \& Shadomy, H.J. (eds) Manual of Clinical Microbiology. American Society for Microbiology, Washington D.C., 1985, pp. 129-137.

29. Updegraff, D.M. A cultural method of quantitatively studying the microorganisms in the skin. J Invest Dermatol 1964, 43: 129-137.

30. Meites, S. \& Levitt, M.J. Skin-puncture and blood-collecting techniques for infants. Clin Chem 1979, 25: 183-189.

31. Washington, J.A., II. Comparison of two commercially available media for detection of bacteremia. Appl Microbiol 1971, 22: 604-607.

32. Hall, M., Warren, E. \& Washington, J.A., II. Comparison of two liquid blood culture media containing sodium polyanetholesulfonate: tryptic soy and Columbia. Appl Microbiol 1974, 27: 699-702.

33. Hall, M., Warren, E. \& Washington, J.A., II. Detection of bacteremia with liquid media containing sodium polyanetholsulfonate. Appl Microbiol 1974, 27: 187-191.

34. Hall, M.M., Ilstrup, D.M. \& Washington, J.A., II. Comparison of three blood culture media with tryptic soy broth. $J$ Clin Microbiol 1978, 8: 299-301.

35. Szymczak, E.G., Barr, J.T., Durbin, W.A. \& Goldmann, D.A. Evaluation of blood culture procedures in a pediatric hospital. J Clin Microbiol 1979, 9: 88-92.

36. Kelly, M.T., Fojtasek, M.F., Abbott, T.M. et al. Clinical evaluation of a lysis-centrifugation technique for the detection of septicemia. JAMA 1983, 250: 2185-2188.

37. Callihan, D.R., Migneault, P.C. \& Nolte, F.S. Clinical and bacteriologic evaluation of BACTEC resin-containing blood culture medium. Am J Clin Pathol 1984, 82: 465-469.

38. Carlson, L.G. \& Plorde, J.J. Influence of a blood culture inoculation technique on detection of bacteremia by the BACTEC system. J Clin Microbiol 1982, 16: 590-592.

39. Wilson, J.A., Barratt, A.J., Gray, J. \& Statham, G.B. Comparison of conventional and single bottle system for blood cultures. J Clin Pathol 1988, 41: 679-682. 
40. Sullivan, N.M., Sutter, V.L., Carter, W.T., Attebery, H.R. \& Finegold, S.M. Bacteremia after genitourinary tract manipulation: bacteriological aspects and evaluation of various blood culture systems. Appl Microbiol 1972, 23: $1101-1106$

41. Richet, H., Escande, M.C. \& Lagrange, P.H. Lysis centrifugation and slide blood culture systems for diagnosis of bacteremia in immunocompromised patients. Eur $J$ Clin Microbiol 1987, 6: 64-67.

42. Schmidt, B.K., Kirpalani, H.M., Corey, M. et al. Coagulasenegative staphylococci as true pathogens in newborn infants: a cohort study. Pediatr Infect Dis J 1987, 6: 1026-1031.
43. Gill, V.J., Zierdt, C.H., Wu, T.C. et al. Comparison of lysis-centrifugation with lysis-filtration and a conventional unvented bottle for blood cultures. J Clin Microbiol 1984, 20: 927-932.

44. Yagev, Y., Simu, A., Alkan, M. Introduction of a semiautomated system for blood cultures. Isr J Med Sci 1984, 20: 475 (abstract)

45. Thomson, R.B., Jr, Vanzo, S.J., Henry, N.K., Guenther, K.L. \& Washington, J.A., II. Contamination of cultures processed with the Isolator lysis-centrifugation blood culture tube. J Clin Microbiol 1984, 19: 97-99. 